\title{
Semeadura cruzada, espaçamento entre fileiras e densidade de semeadura influenciando o crescimento e a produtividade de duas cultivares de soja
}

\author{
Crossed rows, row spacing, and seeding rates influence the growth and yield of two soybean cultivars
}

\section{Alvadi Antonio Balbinot Junior ${ }^{1 *}$, Sérgio de Oliveira Procópio ${ }^{2}$, Norman Neumaier ${ }^{1}$, André Sampaio Ferreira $^{3}$, Flávia Werner ${ }^{3}$, Henrique Debiasi ${ }^{1}$ e Julio Cezar Franchini ${ }^{1}$}

Recebido em 01/07/2015 / Aceito em 03/06/2016

\section{RESUMO}

O objetivo deste trabalho foi avaliar o crescimento de duas cultivares de soja com tipos de crescimento determinado e indeterminado, submetidas a diferentes arranjos espaciais de plantas, incluindo a semeadura cruzada. Foram conduzidos dois experimentos, um com a cultivar BRS 294 RR, que possui tipo de crescimento determinado e grupo de maturidade relativa 6.3 e outro com a cultivar BRS $359 \mathrm{RR}$, tipo de crescimento indeterminado e grupo de maturidade relativa 6.0. Nos dois experimentos, foi utilizado o delineamento experimental de blocos completos ao acaso, em esquema fatorial $2 \times 2 \times 2$, com três repetições. Os tratamentos foram formados pela combinação de dois espaçamentos entrefileiras $(0,4$ e $0,6 \mathrm{~m}$ ), duas densidades de semeadura (375 e 562,5 mil sementes ha-1, com percentual de germinação de $81 \%$ ) e de duas formas de semeadura (semeadura cruzada e não cruzada). O efeito do arranjo de plantas sobre o crescimento foi distinto entre as cultivares. Para a cultivar BRS 294 RR, que possui crescimento vegetativo vigoroso, com alta ramificação, em geral, o aumento da densidade, a redução do espaçamento e o cruzamento das fileiras não propiciou maior crescimento de plantas. No entanto, a maior produtividade foi observada no espaçamento de 0,6 $\mathrm{m}$, em relação ao de $0,4 \mathrm{~m}$. Para a cultivar BRS 359 $\mathrm{RR}$, que possui arquitetura compacta das plantas, o aumento da densidade e a redução do espaçamento propiciaram maior crescimento e cobertura do solo pela comunidade de plantas, mas não afetaram a produtividade de grãos. Nas duas cultivares, a semeadura cruzada não conferiu aumentos de produtividade.
PALAVRAS-CHAVE: tipo de crescimento, arranjo de plantas, índice de área foliar, Glycine max L.

\section{ABSTRACT}

This research aims to evaluate the growth of two soybean cultivars with determinate and indeterminate growth habits under different spatial plant arrangements, including crossed rows. Two experiments were conducted, one with BRS 294 RR cultivar, which has determinate growth habit and relative maturity of 6.3 and another with BRS 359 RR, which has indeterminate growth habit and relative maturity of 6.0. In both experiments, the randomized complete block design in a $2 \times 2 \times 2$ factorial design with three replications was used. The treatments were formed by two row spacing $(0.4$ and $0.6 \mathrm{~m})$, two seeding rates $(375,000$ and 562,500 seeds ha $^{-1}$, with $81 \%$ germination) and two forms of sowing (crossed and uncrossed rows). The effect of plant arrangement on the soybean growth was different between cultivars. In general, for BRS 294 RR, vigorous vegetative growth and high branching, the growth of plant community was not affected by the spatial plant arrangement. However, the highest yield was observed in the $0.6 \mathrm{~m}$ row spacing. For BRS 359 $\mathrm{RR}$, which has compact plant architecture, the highest plant density and the lowest row spacing promoted the growth and the soil cover by plant community, but did not affect the grain yield. In both cultivars, the soybean grain yield was not affected by crossed rows.

KEYWORDS: growth habit, plant arrangement, leaf area index, Glycine max L.

${ }^{1}$ Empresa Brasileira de Pesquisa Agropecuária, Londrina, PR, Brasil.

${ }^{2}$ Empresa Brasileira de Pesquisa Agropecuária, Aracajú, SE, Brasil.

${ }^{3}$ Universidade Estadual de Londrina, Londrina, PR, Brasil.

*Autor para correspondência <alvadi.balbinot@embrapa.br> 


\section{INTRODUÇÃO}

$\mathrm{Na}$ última década, a sojicultura brasileira experimentou mudanças com a utilização de novas tecnologias, como o sistema plantio direto, o advento das cultivares transgênicas e a introdução de cultivares mais produtivas. Entretanto, as novas cultivares de soja apresentam tipo de crescimento e porte diferentes das cultivares usadas até a década de 1990 (TREZZI et al. 2013), promovendo discussões acerca de arranjos espaciais de plantas que maximizem a produtividade de grãos (SOUZA et al. 2010). Diante das novas cultivares e tecnologias de cultivo, faz-se necessário o estudo de ações de manejo que possam refletir em aumento da produtividade, sem grandes mudanças nos custos de produção.

Recentemente, alguns agricultores vêm testando uma forma de semeadura denominada "semeadura cruzada", na qual se realiza uma operação de semeadura, posicionando metade das sementes, seguida de outra operação similar, no sentido perpendicular à primeira. Alguns recordistas de produtividade têm atribuído, pelo menos em parte, a alta produtividade de grãos à semeadura cruzada (PROCÓPIO et al. 2013). Em trabalho desenvolvido por BALBINOT JUNIOR et al. (2015), constatou-se que a semeadura cruzada não promoveu aumento de produtividade de grãos em relação ao espaçamento convencional de $0,45 \mathrm{~m}$, mas incrementou o custo da semeadura e a mobilização do solo. No entanto, faltam informações científicas sobre os efeitos dessa técnica no crescimento das plantas e na produtividade da soja e a sua relação com cultivares e outras práticas de manejo.

O arranjo espacial de plantas, determinado pelo espaçamento entrefileiras e pela densidade de plantas, afeta a competição intraespecífica e, consequentemente, a quantidade de recursos do ambiente (água, luz e nutrientes) disponíveis para cada indivíduo, podendo influenciar a produtividade de grãos (RAMBO et al. 2004; BRUIN \& PEDERSEN 2008; HANNA et al. 2008; COX et al. 2010; WALKER et al. 2010; BOARD \& KAHLON 2013). Além disso, pode afetar a velocidade de fechamento entre as fileiras (HEIFFIG et al. 2006; SILVA et al. 2013), a produção de fitomassa e cobertura do solo pelas plantas (COX \& CHERNEY 2011), a arquitetura das plantas (PIRES et al. 1998), a severidade de doenças (LIMA et al. 2012) e o acamamento (BALBINOT JUNIOR 2011).
Várias pesquisas têm mostrado a pequena resposta da soja às variações de densidade de plantas (PIRES et al. 1998; HEIFFIG et al. 2006; BOARD \& KAHLON 2013). Esse resultado é atribuído à alta plasticidade fenotípica da cultura, a qual é definida como a capacidade da planta alterar sua morfologia e componentes de rendimento a fim de adequálos às condições impostas pelo arranjo espacial dos indivíduos (CESA 1994). Em relação ao efeito do espaçamento entre as fileiras, há resultados discrepantes na literatura (RAMBO et al. 2003; HEIFFIG et al. 2006), pois essa resposta tem forte dependência das cultivares e do ambiente de cultivo. Nesse sentido, a escolha do arranjo espacial de plantas na área deve levar em consideração as características das cultivares utilizadas (EDWARDS \& PURCELL 2005; GASPAR \& CONLEY 2015; THOMPSON et al. 2015).

O crescimento das plantas pode ser caracterizado por diversas variáveis, tais como a massa seca, área foliar, altura de plantas e porcentagem de cobertura do solo pelas estruturas da parte aérea das plantas. Adicionalmente, pode-se estimar o fechamento das entrefileiras com uso do aparelho Green Seeker ${ }^{\circledR} 505$ Handheld Sensor, que é portátil e é equipado com um sensor ativo e não imageador capaz de calcular o Índice de Vegetação por Diferença Normalizada (NDVI). Com o aumento da cobertura vegetal há incremento do NDVI (LIRA et al. 2009).

O objetivo deste trabalho foi avaliar o crescimento de duas cultivares de soja com tipo de crescimento determinado e indeterminado, submetidas a diferentes arranjos espaciais de plantas, incluindo a semeadura cruzada.

\section{MATERIAL E MÉTODOS}

Foram conduzidos dois experimentos em campo, cujas coordenadas geoprocessadas são: latitude $23^{\circ} 11^{\prime}$ sul, longitude $51^{\circ} 11^{\prime}$ oeste e $620 \mathrm{~m}$ de altitude. $\mathrm{O}$ solo da área experimental foi identificado como Latossolo Vermelho distroférrico (SANTOS et al. 2006) e apresentava os seguintes atributos, na camada de 0 a $20 \mathrm{~cm}$, antes da implantação do experimento: $21,4 \mathrm{~g} \mathrm{dm}^{-3}$ de matéria orgânica; 4,9 de $\mathrm{pH} \mathrm{em} \mathrm{CaCl}_{2} ; 8,6 \mathrm{mg} \mathrm{dm}^{-3}$ de P; $0,55 \mathrm{cmol}_{\mathrm{c}} \mathrm{dm}^{-3} \mathrm{de} \mathrm{K}$; 3,7 $\mathrm{cmol}_{\mathrm{c}} \mathrm{dm}^{-3} \mathrm{de} \mathrm{Ca} ; 1,4 \mathrm{cmol}_{\mathrm{c}} \mathrm{dm}^{-3} \mathrm{de} \mathrm{Mg}$; e $48 \%$ de saturação da CTC por bases.

A aveia-preta presente na área experimental foi manejada mecanicamente aos 11 dias antes da 
semeadura da soja, utilizando-se um triturador de restos culturais (triton). Nove dias após essa operação, a vegetação remanescente na área foi dessecada quimicamente com glyphosate $\left(1.080 \mathrm{~g} \mathrm{ha}^{-1}\right) \mathrm{e}$ carfentrazone-ethyl $\left(30 \mathrm{~g} \mathrm{ha}^{-1}\right)$.

Foram conduzidos dois experimentos, um com a cultivar BRS 294 RR, que possui tipo de crescimento determinado, grupo de maturidade relativa 6.3 e alto crescimento vegetativo e outro com a cultivar BRS 359 RR, tipo de crescimento indeterminado, grupo de maturidade relativa $6.0 \mathrm{e}$ plantas com arquitetura compacta e menos vigorosas que a cultivar BRS 294 RR. Nos experimentos foi utilizado o delineamento experimental de blocos completos ao acaso, em esquema fatorial $2 \times 2 \times 2$, com três repetições. Os tratamentos foram formados pela combinação de dois espaçamentos entrefileiras $(0,4$ e $0,6 \mathrm{~m}$ ), duas densidades de semeadura (375 e $562 \mathrm{mil}$ sementes ha ${ }^{-1}$, com poder germinativo de $81 \%$ ) e de duas formas de semeadura (cruzada e não cruzada). As parcelas mediram $8,0 \mathrm{~m}$ de comprimento por 4,8 $\mathrm{m}$ de largura, totalizando $38,4 \mathrm{~m}^{2}$. A área utilizada nas avaliações foi de $14,4 \mathrm{~m}^{2}$ (6 m de comprimento por 2,4 $\mathrm{m}$ de largura). Para as duas cultivares, a densidade de plantas na colheita variou de 270 a 290 mil plantas ha $^{-1}$ na menor densidade avaliada e de 410 a 440 mil plantas ha ${ }^{-1}$ na maior densidade.

A semeadura foi realizada no dia 19/10/2011, a uma velocidade média de $5 \mathrm{~km} \mathrm{~h}^{-1}$, por meio de uma semeadora-adubadora, marca Semeato, modelo SHM 11/13, equipada com sulcadores do tipo facão guilhotina para o adubo e disco duplo defasado para a semente, e dosadores do tipo disco perfurado com dupla fileira de furos para a semente. As sementes de soja foram tratadas com Vitavax-Thiran 200SC ${ }^{\circledR}$ (150 $\mathrm{mL} 50 \mathrm{~kg}^{-1}$ de sementes; $200 \mathrm{~g} \mathrm{~L}^{-1}$ carboxina $+200 \mathrm{~g}$ $\mathrm{L}^{-1}$ tiram), Co-Mo Platinum ${ }^{\circledR}(2,4 \mathrm{~g} \mathrm{Co}+23,4 \mathrm{~g}$ Mo $50 \mathrm{~kg}^{-1}$ de sementes) e inoculante líquido Gelfix $5^{\circledR}$ (Bradyrhizobium elkanii, $100 \mathrm{~mL} 50 \mathrm{~kg}^{-1}$ de sementes). A adubação de base constou da aplicação a lanço de $600 \mathrm{~kg} \mathrm{ha}^{-1}$ de superfosfato simples. Em cobertura, foram aplicados $250 \mathrm{~kg} \mathrm{ha}^{-1}$ de cloreto de potássio a lanço, 16 dias após a semeadura (DAS). A expectativa de produtividade de grãos foi de $5 \mathrm{t} \mathrm{ha}^{-1}$. O controle de pragas, doenças e plantas daninhas foi efetuado conforme as indicações técnicas para a cultura. Os dados de precipitação pluvial e temperatura média do ar durante o período entre a semeadura e a colheita estão apresentados na Figura 1.

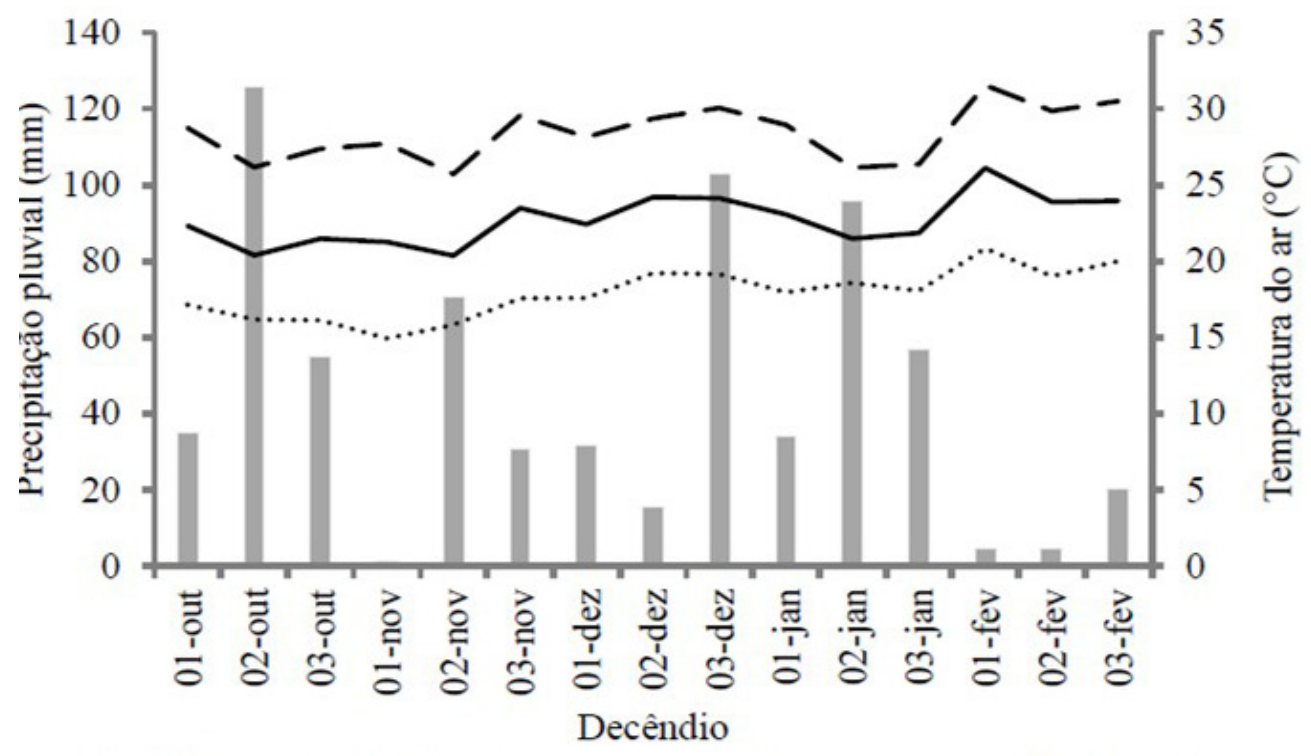

\footnotetext{
- Precipitação pluvial $(\mathrm{mm})(\mathrm{mm})$ - Temperatura média do ar $\left({ }^{\circ} \mathrm{C}\right)$

- - Temperatura máxima do ar $\left({ }^{\circ} \mathrm{C}\right) \quad \cdots \cdots \cdot$.... Temperatura mínima do ar $\left({ }^{\circ} \mathrm{C}\right)$
}

Figura 1 - Precipitação pluvial acumulada por decêndio $(\mathrm{mm})$ e temperaturas máxima, média e mínima do ar $\left({ }^{\circ} \mathrm{C}\right)$ durante o ciclo de desenvolvimento da cultura da soja. Londrina, PR.

Figure 1 - Rainfall (mm), maximum, mean and minimum air temperature $\left({ }^{\circ} \mathrm{C}\right)$ during the soybean crop development. Londrina, Paraná State, Brazil. 
Foram realizadas as seguintes avaliações: 1) Massa seca da parte aérea aos 85 DAS (R5), amostrando-se 10 plantas ao acaso por parcela, sendo a massa por $\mathrm{m}^{2}$ estimada com base na densidade de plantas presente em cada parcela; 2) Índice de área foliar aos 85 DAS, medido em 10 plantas por parcela com auxílio de um integrador de área foliar de bancada; 3) Altura de plantas aos 36 (V4), 50 (V6) e 63 (R2) DAS, determinada em 15 plantas por parcela; 4) Porcentagem da cobertura do solo com plantas, palha e solo exposto aos 28 (V3), 36 (V4), 44 (V5) e 49 (V6) DAS. Para a avaliação da cobertura do solo foi utilizado um aparato que consistia de um perfilado de seção retangular $(2,5 \mathrm{~cm}$ x 5,0 cm) em duralumínio com 2,3 m de altura, e braço horizontal de $1,5 \mathrm{~m}$, cruzando-se num ângulo reto a $20 \mathrm{~cm}$, ficando o comprimento útil do braço em 1,3 m e altura útil em 2,1 m. Para que o conjunto fosse colocado no prumo, foram usados dois níveis afixados com lacres plásticos em duas faces, perpendiculares entre si. Esses níveis ficavam à altura dos olhos do operador, para que, ao visualizar simultaneamente ambos os níveis, pudesse aprumar o sistema na posição correta para fotografar. $\mathrm{Na}$ extremidade do braço horizontal do conjunto foi afixada uma câmera fotográfica (Sony DSC-H9), disparada à distância por meio de controle remoto. $\mathrm{O}$ display articulado de LCD da câmera permitia que o operador do controle remoto visualizasse a imagem antes de fotografar. Em cada parcela foi selecionado um único local em todas as avaliações para o posicionamento da haste vertical do aparato. Em cada foto, por meio do software $\mathrm{AfSoft}^{\circledR}$, desenvolvido pela Embrapa Instrumentação Agropecuária, foi verificada a porcentagem de: a) cobertura do solo pelas plantas de soja; b) cobertura do solo com palha e; c) solo exposto (sem nenhuma cobertura); 5) NDVI aos 60 (R2), 76 (R5) e 84 DAS (R5), utilizando-se o equipamento Green Seeker ${ }^{\circledR} 505$ Handheld Sensor. O NDVI consiste no cálculo da diferença entre emissão e reflexão de dois comprimentos de onda do espectro eletromagnético: infravermelho próximo $(0,725-1,1$ $\mu \mathrm{m})$ e vermelho $(0,58-0,68 \mu \mathrm{m})$, e seu valor varia de -1 a 1 , de acordo com a fórmula: NDVI= $(\rho i v \rho-\rho v) /$ ( $\rho i v \rho+\rho v)$, onde $\rho i v \rho$ é a refletância no infravermelho próximo; $\rho v$ é a refletância no vermelho; 6) $\mathrm{Na}$ fase de plantas maduras foi feita a avaliação do grau de acamamento das plantas, em uma escala de notas de 1 a 5, conforme descrição a seguir: a) nenhuma planta da área útil acamada; b) 25\% das plantas da área útil acamadas; c) 50\% das plantas da área útil acamadas d) $75 \%$ das plantas da área útil acamadas; e) 100\% das plantas da área útil acamadas; 7) Produtividade de grãos, avaliada por meio da colheita das plantas presentes na área útil das parcelas $\left(14,4 \mathrm{~m}^{2}\right)$, sendo os dados convertidos para hectare, na umidade padrão de $13 \%$.

Ajustaram-se equações lineares para obtenção dos coeficientes angulares da evolução da altura de plantas, de 36 a 63 DAS e da cobertura do solo com plantas, de 28 a 49 DAS. Com isso, as três variáveis relacionadas à altura e às quatro relacionadas à porcentagem de cobertura com plantas foram resumidas em apenas dois coeficientes angulares. Todos os dados foram submetidos à análise de variância e teste $F(p \leq 0,05)$. Para a realização da análise estatística, foi utilizado o programa Sisvar (FERREIRA 2011).

\section{RESULTADOS E DISCUSSÃO}

A análise de variância para as duas cultivares está apresentada na Tabela 1. As variáveis coeficiente angular da altura de plantas (AP), NDVI aos 76 DAS e NDVI aos 84 DAS não foram afetadas pelos fatores experimentais, em ambas as cultivares. Na cultivar BRS 359 RR, o NDVI aos 60 DAS e a produtividade também não foram afetados pelos tratamentos. Adicionalmente, não houve acamamento de plantas (nota 1) em todas as parcelas, em razão das cultivares utilizadas possuírem características que lhes conferem reduzido acamamento. A maioria das cultivares de soja lançadas recentemente no mercado brasileiro possuem baixa susceptibilidade ao acamamento (BALBINOT JUNIOR 2011). Nesse caso, o ajuste no arranjo de plantas é focado principalmente no aumento da produtividade, sem considerar o acamamento (COX \& CHERNEY 2011). Estes resultados concordam com os obtidos por LINZMEYER JR. et al. (2008), que, trabalhando com a cultivar CD 208 de tipo de crescimento determinado, não verificaram diferenças no acamamento das plantas de soja com o aumento na população de plantas, de 14 para 18 plantas por metro, utilizando espaçamento de $0,45 \mathrm{~m}$ entre as fileiras.

Na cultivar BRS 294 RR, a massa seca da parte aérea (MSPA) e o índice de área foliar (IAF), ambos medidos aos 85 DAS, bem como o NDVI medido aos 60 DAS foram influenciados pela interação entre a densidade de semeadura e o espaçamento entrefileiras (Tabela 2). O aumento da densidade de semeadura proporcionou diminuição na MSPA e IAF somente no 
Tabela 1 - Análise de variância para a massa seca da parte aérea aos 85 dias após a semeadura - DAS (MSPA), coeficiente angular da altura de plantas dos 36 aos 63 DAS (AP), índice de área foliar aos 85 DAS (IAF), coeficiente angular da cobertura do solo por plantas dos 28 aos 49 DAS (CSPL), cobertura do solo com palha aos 28 DAS (CSPA), solo exposto aos 28 DAS (SE), índice de vegetação por diferença normalizada (NDVI) e produtividade de grãos (PG), avaliadas em experimentos conduzidos com as cultivares de soja BRS 294 RR e BRS 359 RR.

Table 1 - Variance analysis for dry weight of shoot after 85 days after sowing - DAS (MSPA), angular coefficient of plant height of from 36 to $63 \mathrm{DAS}(A P)$, leaf area index at $85 \mathrm{DAS}$ (IAF), angular coefficient of soil cover by plants from 28 to 49 DAS (CSPL), soil cover with straw at 28 DAS (CSPA), bare soil at 28 DAS (SE), Normalized Difference Vegetation Index (NDVI), and grain yield (PG), evaluated in experiments carried out with the soybean cultivars BRS 294 RR and BRS 359 RR.

\begin{tabular}{|c|c|c|c|c|c|c|c|c|c|c|}
\hline \multirow{4}{*}{ Causas de Variação } & \multicolumn{2}{|c|}{ MSPA } & \multicolumn{2}{|c|}{ AP } & \multicolumn{2}{|c|}{ IAF } & \multicolumn{2}{|c|}{ CSPL } & \multicolumn{2}{|c|}{ CSPA } \\
\hline & BRS & BRS & BRS & BRS & BRS & BRS & BRS & BRS & BRS & BRS \\
\hline & 294 & 359 & 294 & 359 & 294 & 359 & 294 & 359 & 294 & 359 \\
\hline & $\mathrm{RR}$ & $\mathrm{RR}$ & RR & $\mathrm{RR}$ & $\mathrm{RR}$ & $\mathrm{RR}$ & RR & $\mathrm{RR}$ & $\mathrm{RR}$ & $\mathrm{RR}$ \\
\hline Espaçamento (E) & ns & ns & ns & ns & ns & ns & ns & $* *$ & ns & ns \\
\hline Densidade (D) & $\mathrm{ns}$ & $* *$ & $\mathrm{~ns}$ & $\mathrm{~ns}$ & $\mathrm{~ns}$ & $*$ & $\mathrm{~ns}$ & $\mathrm{~ns}$ & $* *$ & $*$ \\
\hline Cruzamento (C) & $\mathrm{ns}$ & $* *$ & $\mathrm{~ns}$ & $\mathrm{~ns}$ & $\mathrm{~ns}$ & $* *$ & $\mathrm{~ns}$ & $\mathrm{~ns}$ & $\mathrm{~ns}$ & $*$ \\
\hline$E \times D$ & $* *$ & ns & ns & ns & $*$ & ns & ns & ns & ns & ns \\
\hline $\mathrm{E} \times \mathrm{C}$ & ns & ns & ns & ns & ns & ns & $* *$ & $* *$ & ns & ns \\
\hline $\mathrm{D} \times \mathrm{C}$ & ns & ns & $\mathrm{ns}$ & $\mathrm{ns}$ & $\mathrm{ns}$ & ns & * & ns & ns & ns \\
\hline \multirow[t]{2}{*}{$\mathrm{E} \times \mathrm{D} \times \mathrm{C}$} & ns & ns & ns & ns & $\mathrm{ns}$ & ns & ns & ns & ns & ns \\
\hline & \multicolumn{2}{|c|}{ SE } & \multicolumn{2}{|c|}{ NDVI 60 DAS } & \multicolumn{2}{|c|}{ NDVI 76 DAS } & \multicolumn{2}{|c|}{ NDVI 84 DAS } & \multicolumn{2}{|c|}{ PG } \\
\hline Espaçamento (E) & ns & ns & ns & ns & ns & ns & ns & ns & $* *$ & ns \\
\hline Densidade (D) & ns & ns & $\mathrm{ns}$ & ns & ns & ns & ns & ns & ns & ns \\
\hline Cruzamento (C) & $*$ & $* *$ & $\mathrm{~ns}$ & $\mathrm{~ns}$ & $\mathrm{~ns}$ & ns & $\mathrm{ns}$ & $\mathrm{ns}$ & $\mathrm{ns}$ & $\mathrm{ns}$ \\
\hline$E \times D$ & $\mathrm{~ns}$ & $\mathrm{~ns}$ & $*$ & $\mathrm{~ns}$ & $\mathrm{~ns}$ & ns & $\mathrm{ns}$ & ns & $\mathrm{ns}$ & ns \\
\hline $\mathrm{E} \times \mathrm{C}$ & ns & $* *$ & ns & ns & ns & ns & ns & ns & ns & ns \\
\hline $\mathrm{D} \times \mathrm{C}$ & ns & ns & ns & ns & ns & ns & ns & ns & ns & $\mathrm{ns}$ \\
\hline$E \times D \times C$ & ns & ns & ns & ns & ns & $\mathrm{ns}$ & ns & ns & ns & ns \\
\hline
\end{tabular}

ns $=$ Não significativo a $5 \%$ de probabilidade pelo teste $\mathrm{F} . *$ Significativo a $5 \%$ de probabilidade pelo teste $\mathrm{F}$.

** Significativo a $1 \%$ de probabilidade pelo teste $\mathrm{F}$.

espaçamento mais amplo $(0,6 \mathrm{~m})$. Por outro lado, o IAF não foi afetado pelo espaçamento e a MSPA foi superior no maior espaçamento e na menor densidade avaliada. Isso demonstra que, para a cultivar BRS 294 $\mathrm{RR}$, a melhor condição para crescimento foi obtida com espaçamento de $0,6 \mathrm{~m}$ e densidade de $375 \mathrm{mil}$ sementes $\mathrm{ha}^{-1}$, provavelmente por esse genótipo apresentar alta capacidade de ramificação e ocupação do espaço. Quando se utilizou a maior densidade de semeadura $\left(562,5\right.$ mil sementes ha $\left.{ }^{-1}\right)$ foi constatado que o maior espaçamento $(0,6 \mathrm{~m})$ promoveu um maior valor de NDVI em relação a $0,4 \mathrm{~m}$, avaliado aos 60 DAS (Tabela 2). Nesse trabalho não houve correlação do NDVI medido aos 60, 76 e 84 DAS com a MSPA e IAF aos 85 DAS, em ambas as cultivares. Isso ocorreu porque o NDVI indica a cobertura do solo pelas plantas, desconsiderando a estrutura do dossel. Todavia, em trabalhos desenvolvidos por DORAISWAMY et al. (2004), DORAISWAMY et al. (2005) e RIZZI \& RUDORFF (2007) foram detectadas correlações significativas e positivas entre IAF e NDVI no início do ciclo de desenvolvimento.

Na cultivar BRS 359 RR, a MSPA e o IAF, avaliados aos 85 DAS, foram maiores na densidade mais elevada e na disposição não cruzada das fileiras de semeadura (Tabela 3 ). 
Tabela 2 - Massa seca da parte aérea aos 85 dias após a semeadura (DAS), índice de área foliar aos 85 DAS e NDVI aos 60 DAS de plantas de soja, em diferentes espaçamentos e densidades de semeadura de soja, cultivar BRS 294 RR.

Table 2 - Shoot dry mass at 85 days after sowing (DAS), leaf area index at 85 DAS and NDVI at 60 DAS of soybean plants in different spacing and soybean seeding rates, BRS 294 RR.

\begin{tabular}{|c|c|c|}
\hline \multirow[t]{2}{*}{ Espaçamento (m) } & \multicolumn{2}{|c|}{ Densidade de semeadura (mil sementes $\mathrm{ha}^{-1}$ ) } \\
\hline & 375 & 562,5 \\
\hline & \multicolumn{2}{|c|}{ Massa seca da parte aérea aos 85 DAS $\left(\mathrm{g} \mathrm{m}^{-2}\right)$} \\
\hline 0,4 & $531 \mathrm{a}^{1} \mathrm{~B}^{1}$ & $583 \mathrm{aA}$ \\
\hline 0,6 & $634 \mathrm{aA}$ & $481 \mathrm{bA}$ \\
\hline CV $(\%)$ & \multicolumn{2}{|c|}{15,0} \\
\hline & \multicolumn{2}{|c|}{ Índice de área foliar aos 85 DAS } \\
\hline 0,4 & $5,4 \mathrm{aA}$ & $6,1 \mathrm{aA}$ \\
\hline 0,6 & $6,6 \mathrm{aA}$ & $5,2 \mathrm{bA}$ \\
\hline CV $(\%)$ & \multicolumn{2}{|c|}{16,7} \\
\hline & \multicolumn{2}{|c|}{ NDVI aos 60 DAS } \\
\hline 0,4 & $0,44 \mathrm{aA}$ & $0,40 \mathrm{aB}$ \\
\hline 0,6 & $0,41 \mathrm{aA}$ & $0,51 \mathrm{aA}$ \\
\hline $\mathrm{CV}(\%)$ & \multicolumn{2}{|c|}{18,3} \\
\hline
\end{tabular}

Tabela 3 - Massa seca da parte aérea aos 85 dias após a semeadura - DAS (MSPA), índice de área foliar aos 85 DAS (IAF) e cobertura do solo com palha aos 28 DAS (CSPA) em diferentes densidades de semeadura e cruzamento das fileiras de soja, cultivar BRS 359 RR.

Table 3 - Shoot dry mass at 85 days after sowing - DAS (MSPA), leaf area index at 85 DAS (IAF) and soil cover with straw at 28 DAS (CSPA) at different sowing rates and crossing of soybean lines, BRS 359 RR.

\begin{tabular}{|c|c|c|c|c|}
\hline & \multicolumn{2}{|c|}{$\begin{array}{l}\text { Densidade de semeadura } \\
\quad\left(\text { mil sementes } \text { ha }^{-1}\right)\end{array}$} & \multicolumn{2}{|c|}{ Cruzamento das fileiras } \\
\hline & 375 & 562,5 & Não Cruzado & Cruzado \\
\hline $\operatorname{MSPA}\left(\mathrm{g} \mathrm{m}^{-2}\right)$ & $431 \mathrm{~b}^{1}$ & $520 \mathrm{a}$ & $525, \mathrm{a}$ & $426 \mathrm{~b}$ \\
\hline CV $(\%)$ & & & & \\
\hline IAF & $4,5 \mathrm{~b}$ & $5,3 \mathrm{a}$ & $5,5 \mathrm{a}$ & $4,4 \mathrm{~b}$ \\
\hline CV $(\%)$ & & & & \\
\hline CSPA $(\%)$ & $64,1 \mathrm{a}$ & $60,3 \mathrm{~b}$ & $64,1 \mathrm{a}$ & $60,3 \mathrm{~b}$ \\
\hline $\mathrm{CV}(\%)$ & & & & \\
\hline
\end{tabular}

O espaçamento entre as fileiras não afetou essas duas variáveis (Tabela 1). Segundo COX \& CHERNEY (2011), em cultivares que apresentam plantas compactas, como é o caso da BRS 359 RR, a redução do espaçamento entrefileiras pode conferir maior interceptação de luz no início do ciclo de desenvolvimento, proporcionado maior acúmulo de MSPA e IAF, o que pode refletir em aumento da produtividade de grãos. A redução da MSPA acumulada na semeadura cruzada pode ser creditada a maior mobilização do solo ocasionada pela segunda passagem da semeadora e pela compactação adicional do solo imposta pelos rodados do trator e da semeadora.

A cobertura do solo com palha aos 28 DAS, na cultivar BRS 359 RR, foi maior na menor densidade, 
em razão da menor cobertura com plantas, e no tratamento não cruzado em relação ao cruzado (Tabela 3 ). Isso ocorreu porque com o cruzamento das fileiras houve maior mobilização do solo, promovendo a retirada parcial da palha sobre este. Em ambas as cultivares, contatou-se associação significativa entre IAF e MSPA (Figura 2). Ou seja, é possível estimar o IAF por meio de dados de MSPA, já que a estimativa de área foliar depende do integrador de área foliar, nem sempre disponível nas instituições de pesquisa. Por outro lado, se houver possibilidade de realizar a estimativa de IAF com equipamento portátil, em campo, é possível estimar a MSPA, evitando a destruição das plantas. Adicionalmente, verifica-se que houve incremento de 0,009 e 0,010 no IAF para cada $\mathrm{g} \mathrm{m}^{-2}$ de aumento da massa seca da parte aérea aos 85 DAS para as cultivares BRS 294 RR e BRS $359 \mathrm{RR}$, respectivamente. Ou seja, apesar das grandes diferenças na arquitetura de plantas, a relação entre a massa da parte aérea e o IAF foi semelhante entre as cultivares.
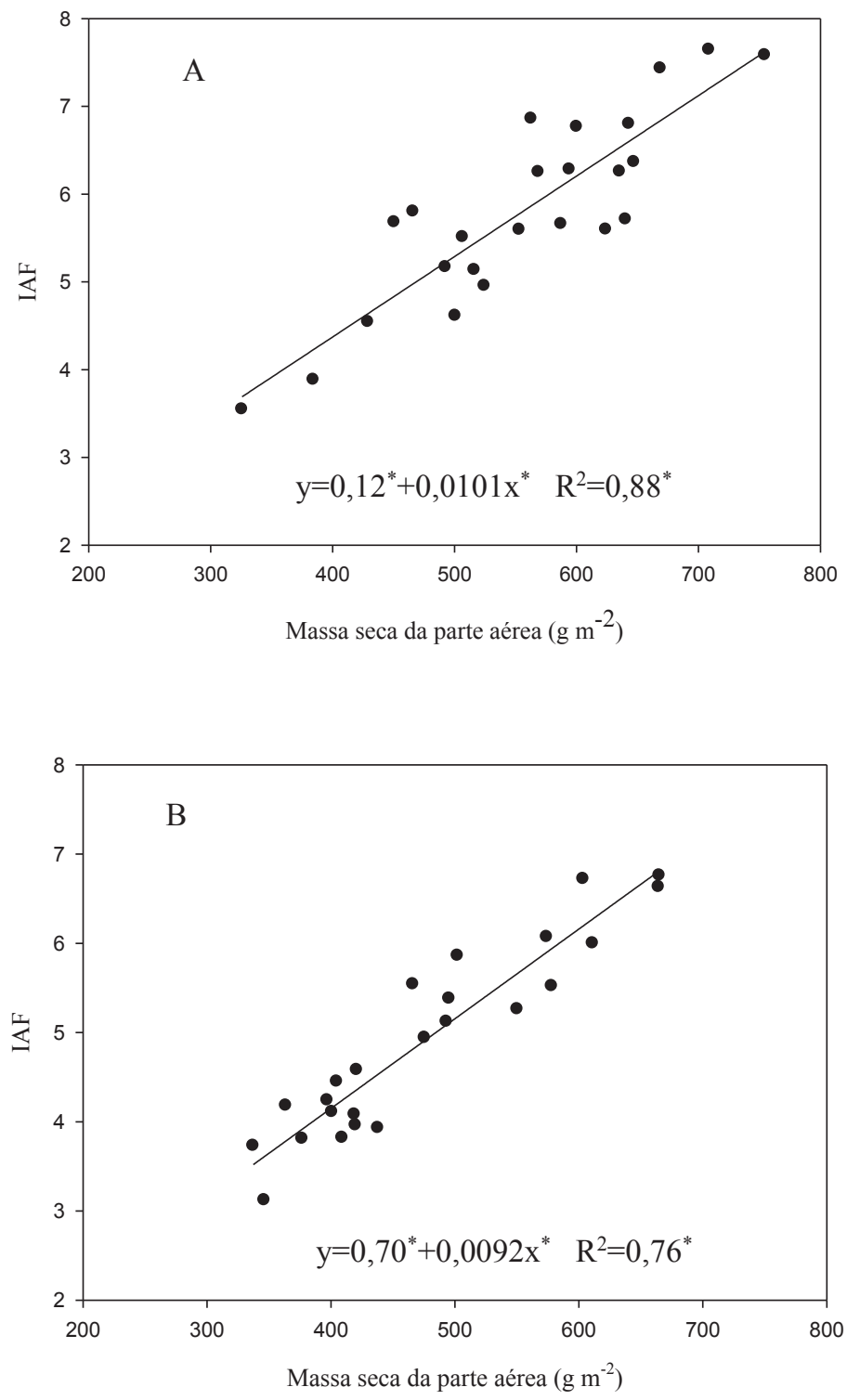

Figura 2 - Relação entre massa seca da parte aérea das plantas de soja e índice de área foliar (IAF) medidos aos 85 dias após a semeadura da cultivar BRS 294 RR (A) e BRS 359 RR (B). *Coeficientes significativos a $5 \%$ de probabilidade.

Figure 2 - Relationship between dry weight of shoot and leaf area index (IAF) of the soybean plants measured at 85 days after sowing the cultivar BRS $294 R R(A)$ and BRS $359 R R$ (B). ${ }^{*}$ Coefficients significant at $5 \%$ probability. 
$\mathrm{Na}$ cultivar BRS 294 RR, o coeficiente angular do modelo linear ajustado para a evolução da cobertura do solo pelas plantas de soja entre 28 e 49 DAS foi superior no espaçamento mais estreito em relação a $0,6 \mathrm{~m}$, tanto na semeadura cruzada como na não cruzada (Tabela 4). SILVA et al. (2013), também verificaram maior fechamento das entrefileiras das cultivares de soja M-Soy 9144 RR e P99R01 quando foram utilizados espaçamentos menores que 0,5 m. Também se verificou que a semeadura cruzada proporcionou fechamento mais rápido do dossel quando foram semeadas 375 mil sementes ha ${ }^{-1}$ de soja (Tabela 4). Na cultivar BRS 359 RR, o comportamento para essa variável foi similar à cultivar BRS 294 RR, em que a semeadura cruzada apresentou maior taxa de cobertura do solo pelas plantas de soja somente no maior espaçamento entrefileiras, contudo o espaçamento de $0,4 \mathrm{~m}$ não diferiu do $0,6 \mathrm{~m}$ quando se cruzou as fileiras de semeadura (Tabela 5). Entre 28 e 49 DAS, a taxa diária de cobertura do solo pelas plantas da cultivar BRS 294 RR foi de 3,45\% e para a cultivar BRS 359 RR foi de 3,00\%, demonstrando a capacidade diferenciada dessas duas cultivares em cobrir o solo com seu dossel.

A semeadura cruzada conferiu maior porcentagem de solo exposto aos $28 \mathrm{DAS}$ em ambas as cultivares (Tabelas 5 e 6). Isso ocorreu porque houve duas operações de semeadura e, consequentemente, maior mobilização do solo, o que pode aumentar a emergência de plantas daninhas fotoblásticas positivas, a perda de água por evaporação e a erosão hídrica.

$\mathrm{Na}$ cultivar BRS $359 \mathrm{RR}$, os tratamentos não afetaram a produtividade de grãos (Tabela 1). $\mathrm{Na}$ cultivar BRS $294 \mathrm{RR}$, apenas o fator espaçamento influenciou na produtividade, sendo que o espaçamento mais amplo proporcionou maior produtividade de grãos (Tabela 6). É provável que isso tenha ocorrido pela maior penetração de luz no dossel no maior espaçamento entrefileiras, refletindose em maior fotossíntese das folhas inferiores e maior acúmulo de massa seca por planta, principalmente na menor densidade de semeadura (Tabela 2), com reflexos positivos na produtividade. Além disso, o maior espaçamento testado possivelmente permitiu maior penetração de inseticidas e fungicidas no dossel, proporcionando maior controle de pragas e doenças nas folhas mais próximas ao solo (MADALOSSO et al. 2010). THOMAS et al. (1998) observaram que, em adequada disponibilidade de nutrientes, houve maior produtividade de grãos de soja em espaçamento entrefileiras de $0,4 \mathrm{~m}$ em relação a $0,2 \mathrm{~m}$, concordando com os resultados obtidos na cultivar BRS 294 RR. Os resultados da presente pesquisa indicaram ausência de aumento de produtividade com a semeadura cruzada. Por outro lado, em pesquisa conduzida por LIMA et al. (2012) no Mato Grosso do Sul, constatou-se aumento da produtividade com o uso dessa técnica, muito provavelmente em razão do uso de cultivares com baixa capacidade de ramificação, em que as plantas têm menor habilidade para fechar as entrefileiras em semeaduras não cruzadas.

Tabela 4 - Coeficiente angular da cobertura do solo com plantas estimado entre 28 e 49 dias após a semeadura em diferentes espaçamentos e densidade de semeadura, cultivar BRS 294 RR.

Table 4 - Angular coefficient angular of soil cover with plants estimated between 28 and 49 days after sowing in different row spacing and seeding rate, BRS 294 RR.

\begin{tabular}{lcc}
\hline & \multicolumn{1}{c}{ Coeficiente angular da cobertura do solo com plantas $\left(\% \mathrm{dia}^{-1}\right)$} \\
\hline \multirow{2}{*}{ Espaçamento $(\mathrm{m})$} & \multicolumn{2}{c}{ Cruzamento das fileiras } \\
\cline { 2 - 3 } 0,4 & $3,6 \mathrm{a}^{1} \mathrm{~A}^{1}$ & Cruzado \\
\hline 0,6 & $3,0 \mathrm{bB}$ & $3,7 \mathrm{aA}$ \\
& & $3,5 \mathrm{aB}$ \\
\hline $\begin{array}{c}\text { Densidade de semeadura } \\
\left(\text { mil sementes ha }{ }^{-1}\right)\end{array}$ & $3,8 \mathrm{aA}$ \\
375 & $3,4 \mathrm{bA}$ & $3,4 \mathrm{aB}$ \\
562,5 & $3,3 \mathrm{aA}$ & \\
$\mathrm{CV}(\%)$ & 4,2 & \\
& &
\end{tabular}

90 Revista de Ciências Agroveterinárias, Lages, v.15, n.2, 2016 
Tabela 5 - Coeficiente angular da cobertura do solo com plantas estimado entre 28 e 49 dias após a semeadura (DAS) e porcentagem de solo descoberto aos 28 DAS em diferentes espaçamentos e cruzamento das fileiras, cultivar BRS 359 RR.

Table 5 - Angular coefficient of soil cover with plants estimated between 28 and 49 dias after sowing (DAS) and percentage of bare ground to 28 DAS in different spacing and crossing the rows, BRS $359 R R$.

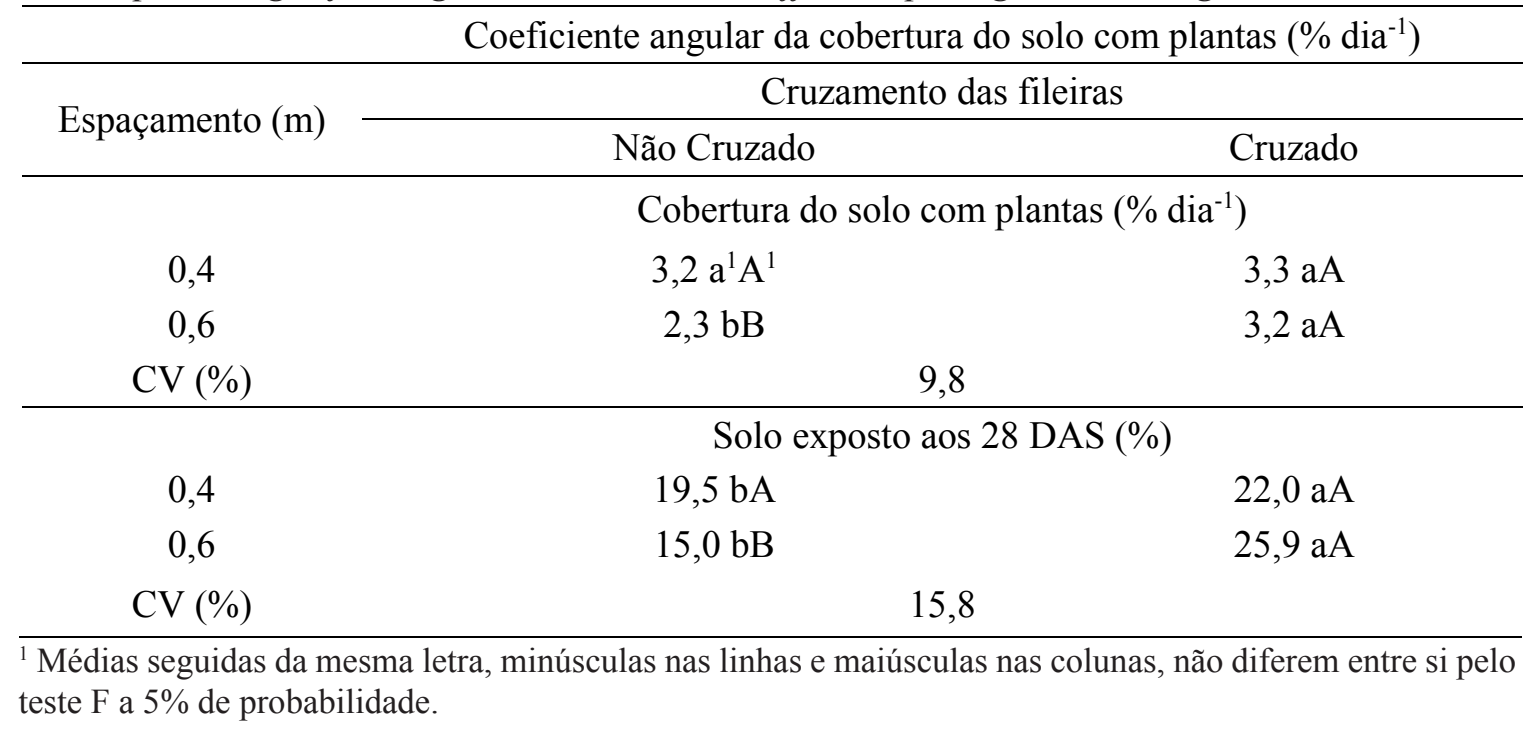

Tabela 6 - Produtividade de grãos, cobertura do solo com palha e solo exposto aos 28 dias após a semeadura (DAS) da cultivar BRS 294 RR em diferentes densidades de semeadura, cruzamento das fileiras e espaçamentos.

Table 6 - Grain yield, soil cover with straw and bare soil at 28 days after sowing (DAS) of BRS 294 RR in different sowing densities, crossing the rows and spacing.

\begin{tabular}{ccc}
\hline & \multicolumn{3}{c}{ Densidade de semeadura (mil sementes ha $\left.{ }^{-1}\right)$} \\
\cline { 2 - 3 } & \multicolumn{3}{c}{375} & 562,5 \\
\cline { 2 - 3 } $\begin{array}{c}\text { Cobertura do solo com } \\
\text { palha aos 28 DAS (\%) } \\
\text { CV (\%) }\end{array}$ & $63,4 \mathrm{a}^{1}$ & $58,5 \mathrm{~b}$ \\
\hline \multicolumn{3}{c}{ Cruzamento das fileiras } \\
\cline { 2 - 3 } Solo exposto aos \\
28 DAS (\%) \\
CV (\%)
\end{tabular}




\section{CONCLUSÕES}

Para a cultivar BRS 294 RR, que possui tipo de crescimento determinado, crescimento vegetativo vigoroso e alta ramificação, o aumento da densidade, a redução do espaçamento e o cruzamento das fileiras, em geral, não propiciaram maior crescimento da comunidade de plantas, sendo que o espaçamento entrefileiras de $0,6 \mathrm{~m}$ conferiu maior produtividade em relação a $0,4 \mathrm{~m}$.

Para a cultivar BRS 359 RR, que possui tipo de crescimento indeterminado e arquitetura compacta de plantas, o aumento da densidade e a redução do espaçamento propiciaram maior crescimento e cobertura do solo pela comunidade de plantas, mas não afetaram a produtividade de grãos.

Nas duas cultivares avaliadas, a semeadura cruzada não aumentou a produtividade de grãos em relação à semeadura sem o cruzamento das fileiras.

\section{REFERÊNCIAS}

BALBINOT JUNIOR AA. 2011. Acamamento de plantas na cultura da soja. Agropecuária Catarinense 25: 40-43.

BALBINOT JUNIOR AA et al. 2015. Espaçamento reduzido e plantio cruzado associados a diferentes densidades de plantas em soja. Semina: Ciências Agrárias 36: 2977-2986.

BOARD JE \& KAHLON CS. 2013. Morphological responses to low plant population differ between soybean genotypes. Crop Science 53: 1109-1119.

BRUIN JL \& PEDERSEN P. 2008. Effect of row spacing and seeding rate on soybean yield. Agronomy Journal 100: 704-710.

COOPERATIVE EXTENSION SERVICE AMES - CESA. 1994. How a soybean plant develops. Ames: Iowa State University of Science and Technology. 20p.

COX WJ et al. 2010. Soybeans compensate at low seeding rates but not at high thinning rates. Agronomy Journal 102: 1238-1243.

COX WJ \& CHERNEY JH. 2011. Growth and yield responses of soybean to row spacing and seeding rate. Agronomy Journal 103: 123-128.

DORAISWAMY PC et al. 2004. Crop condition and yield simulations using Landsat and MODIS. Remote Sensing of Environment 92: 548-559.

DORAISWAMY PC et al. 2005. Application of MODIS derived parameters for regional crop yield assessment. Remote Sensing Environment 97: 192-202.

EDWARDS JT \& PURCELL LC. 2005. Soybean yield and biomass response to increasing plant population among diverse maturity groups. Crop Science 45: 1770-1777.

FERREIRA DF. 2011. SISVAR: um programa para análises e ensino de estatística. Ciência e Agrotecnologia 35: 10391042.

GASPAR AP \& CONLEY SP. 2015. Responses of canopy reflectance, light interception, and soybean seed yield to replanting suboptimal stands. Crop Science 55: 377-385.

HANNA S et al. 2008. Fungicide application timing and row spacing effect on soybean canopy penetration and grain yield. Agronomy Journal 100: 1488-1492.

HEIFFIG LS et al. 2006. Fechamento e índice de área foliar da cultura da soja em diferentes arranjos espaciais. Bragantia 65: 285-295.

LIMA SF et al. 2012. Efeito da semeadura em linhas cruzadas sobre a produtividade de grãos e severidade da ferrugem asiática da soja. Bioscience Journal 28: 954-962. LINZMEYER JR. R et al. 2008. Influência de retardante vegetal e densidades de plantas sobre o crescimento, acamamento e produtividade da soja. Acta Scientiarum Agronomy 30: 373-379.

LIRA VM et al. 2009. Análise espectral de índice de vegetação em área irrigada com cana-de-açúcar. Engenharia Ambiental 6: 113-120.

MADALOSSO MG et al. 2010. Cultivares, espaçamento entrelinhas e programas de aplicação de fungicidas no controle de Phakopsora pachyrhizi Sidow em soja. Ciência Rural 40: 2256-2261.

PIRES JLF et al. 1998. Rendimento de grãos de soja influenciado pelo arranjo de plantas e níveis de adubação. Pesquisa Agropecuária Gaúcha 4: 183-188.

PROCÓPIO SO et al. 2013. Plantio cruzado na cultura da soja utilizando uma cultivar de hábito de crescimento indeterminado. Revista de Ciências Agrárias 56: 319-325.

RAMBO L et al. 2003. Rendimento de grãos da soja em função do arranjo de plantas. Ciência Rural 33: 405-411.

RAMBO L et al. 2004. Estimativa do potencial de rendimento por estrato do dossel da soja, em diferentes arranjos de plantas. Ciência Rural 34: 33-40.

RIZZI R \& RUDORFF BFT. 2007. Imagens do sensor MODIS associadas a um modelo agronômico para estimar a produtividade de soja. Pesquisa Agropecuária Brasileira 42: 73-80.

SANTOS HG et al. 2006. Sistema brasileiro de classificação de solos. 2.ed. Rio de Janeiro: Embrapa Solos. 306p.

SILVA WB et al. 2013. Desenvolvimento inicial de Urochloa ruziziensis e desempenho agronômico da soja em diferentes arranjos espaciais no cerrado Mato-Grossense. Bragantia 72: 146-153.

SOUZA CA et al. 2010. Relação entre densidade de plantas e genótipos de soja Roundup Ready ${ }^{\mathrm{TM}}$. Planta Daninha 28: 887-896.

THOMAS AL et al. 1998. Rendimento de grãos de soja afetado pelo espaçamento entre linhas e fertilidade do solo. Ciência Rural 28: 543-546.

THOMPSON NM et al. 2015. Mid-South soybean yield and net return as affected by plant population and row spacing. Agronomy Journal 107: 979-989. 
Balbinot Junior et al.

TREZZI MM et al. 2013. Competitive ability of soybean cultivars with horseweed (Conyza bonariensis). Planta Daninha 31: 543-550.

WALKER ER et al. 2010. Plant population and rowspacing effects on maturity group III soybean. Agronomy Journal 102: 821-826. 